\title{
USER-FAIR DESIGNING EMERGENCY SERVICE SYSTEMS
}

\author{
Jaroslav JANÁČEK*, Lýdia GÁBRIŠOVÁ \\ Dept of Mathematical Methods and Operations Research, Faculty of Management Science and Informatics, \\ University of Žilina, Slovakia
}

Received 26 February 2016; revised 30 May 2016; accepted 10 July 2016

\begin{abstract}
The usual approach to emergency system design consists in deploying a given number of service centers to minimize the disutility perceived by an average user, what is called "min-sum" or "system approach". As a user in emergency tries to obtain service from the nearest service center, the min-sum optimal deployment may cause such partitioning of the users' set into clusters serviced by one center that population of users is unequally distributed among centers. Within this paper, we focus on user-fair design of emergency service systems, where the fair approach is not applied on the individual users, but on the clusters serviced by one center. The fairer deployment should prevent the users to some extent from frequent occurrence of the situation, when the nearest service center to a current demand location is occupied by servicing some previously raised demand. In such case, the current demand must be assigned to a more distant center. To achieve fairer design of emergency system, we present four approaches to the design problem together with their implementation and comparison using numerical experiments performed with several real-sized benchmarks.
\end{abstract}

Keywords: fair design, emergency service system, location problem, approximate approach, decomposition heuristic technique.

\section{Introduction}

Emergency service systems are designed to satisfy public demand for more secure life. The users - inhabitants of a serviced region are spread over associated geographical area and they are concentrated in a finite set of dwelling places as towns, villages and hamlets.

The emergency service is provided by given number of facilities, which can be deployed in given finite set of possible service center locations in a road network connecting all dwelling places and potential service center locations. Host of emergency service system design approaches are focused on minimizing disutility perceived by an average user. The mentioned disutility is usually proportional to the distance or travelling time from user location to the nearest located service center. This objective leads to the formulation of a weighted $p$-median problem, where number of located facilities is limited by the number $p$ and the weight of user is proportional to the number of inhabitants of the given dwelling place.

Optimal solution of the weighted $p$-median problem minimizes the above mentioned objective of the average user, but this solution creates system of user clusters, where each cluster is serviced by one service center.
This way, the workload of individual facilities located at the centers may considerably differ. This inequality in facility workload causes overloading of some service centers to such extent that the randomly occurring user's demand have to wait until service of the preceding demands has been satisfied. Thus, the waiting time of some exposed users in the region serviced by an overloaded center exceeds the waiting time of the average user several times. Such situation is considered to be unfair in public service systems, where equality in access to service is accented.

Within this paper, we focus on handling this phenomenon and try to suggest and compare four methods of emergency system design following objective to minimize differences among workloads of emergency service system centers. As each attempt at fairer system design is paid by loss of the average user objective, we investigate this effect in connection with the suggested methods. All tackled methods are designed in the way taking into consideration not only their performance (effectivity) as the ratio of computational time and model size, but also requests of a potential designer, which usually needs at hand decision supporting tool software.

${ }^{*}$ Corresponding author. E-mail: jaroslav.janacek@fri.uniza.sk 
The paper is organized as follows: Section 1 gives a brief review of relevant bibliography and Section 2 describes the solved problem by means of mathematical programming together with explanation of involved intricacy. Section 3 presents four studied methods, which comparison is based on results of numerical experiments reported in Section 4. Last section summarizes the obtained findings and gives recommendation for a possible future research.

\section{Literature review}

Public and private service system designs are close connected with location theory and associated development of the optimization techniques. The private service system designs, where minimal total cost is the main objective and the number of located facilities results from tradeoff between cost of user's demand satisfaction and cost of facility locating. The associated model of distribution system design is called uncapacitated facility location problem and effective algorithms were designed in researches by Erlenkotter (1978), Körkel (1989), Janáček and Buzna (2008).

The public service systems (Marianov, Serra 2002; Ingolfsson et al. 2008) including medical emergency system (Doerner et al. 2005; Jánošíková 2007; Brotcorne et al. 2003; Chanta et al. 2014), fire brigade deployment, system of police stations and public administration system (Janáček et al. 2010; Janacek, Kvet 2012; Kvet, Janáček 2015) were usually modelled as a weighted $p$-median problem.

The necessity to solve big instances of the $p$-median problem lead to design of special algorithm (Avella et al. 2007) and also to development of very effective radial formulation of the problem. The radial formulation was used both in construction of special software tool (García et al. 2011) and in suggesting an approximate approach implementable on common commercial Integer Programming (IP)-solver (Janáček 2008; Janacek, Kvet 2014).

The fair design of emergency systems is based on notion of fairness, which accents equality of users in access to service. The fairness was broadly studied in researches by Bertsimas et al. (2011), Marsh and Schilling (1994), Janacek and Kvet (2012), and subsequently many schemes of fairness were suggested. In connection with the schemes various approaches to the fair system design were developed (Ogryczak, Śliwiński 2006; Buzna et al. 2014).

The facility workload equality studied in this paper also corresponds to restricted capacity of service centers. This phenomenon emerging in distribution systems was modeled and some solving techniques were suggested in researches by Pirkul and Schilling (1989), Holmberg et al. (1999), Janáček and Gábrišová (2009), Jánošíková and Žarnay (2014), where the limited capacity and compactness of serviced clusters were studied.

\section{Mathematical model of problem}

The emergency service system considered in this paper provides the users concentrated at dwelling places of a given geographic region with service, which usually mitigates negative impact of randomly arising events. The service of users is provided from the nearest service center. When service centers are deployed, only locations from finite set of candidate locations can be taken into account. Each service center is equipped with one or more facilities, e.g. ambulance vehicles. Contrary to the most of public service system designs, we apply two contradicting objectives on the studied problem. We search for such deployment of $p$ facilities at locations from the set of possible service center locations that the sum of weighted distances from users' locations to the nearest facility is satisfactorily small and simultaneously, the population volumes serviced by individual facilities should differ as little as possible. If only the first one of the objectives is applied, the problem reduces to the weighted $p$-median problem, which solution hardly locates two or more facilities at the same center location. When the second objective is accented, the optimal solution may collapse to the case, where all facilities are located at one center and the users are regularly distributed among the facilities.

To describe the problem by means of mathematical programming, we introduce the following denotation of input data:

- $I$ is the set of candidate center locations with one or more facilities;

- $J$ is the set of municipalities, i.e. users' locations;

- $p$ is the number of facilities to be located;

- $d_{i j}$ is the shortest distance from users' location $j \in J$ to possible center location $i \in I$;

- $b_{j}$ is the number of inhabitants (users) of municipality $j \in J$.

The decision on locating facilities at potential center location $i$ will be modelled by integer variable $y_{i}$, which gives the number of facilities, placed at the location $i$.

Let us introduce following auxiliary denotation, where $Q$ is the set of feasible solution in $|I|$-dimensional space described by constraints (1) and (2):

$$
\begin{aligned}
& \sum_{i \in I} y_{i}=p ; \\
& y_{i} \in Z_{0}^{+} \text {for } i \in I .
\end{aligned}
$$

Then, a mathematical model of the problem with the first objective can be formulated as (3):

$$
\min \left\{\sum_{j \in J} b_{j} \cdot \min \left\{d_{i j}: i \in I, y_{i} \geq 1\right\}: \boldsymbol{y} \in Q\right\} .
$$

Let us define subscript $k(\boldsymbol{y}, j)$ for given solution $\boldsymbol{y} \in Q$ as the subscript of the nearest facility location to users' location $j$. Then, the number $B_{i}(\boldsymbol{y})$ of all users serviced by the center located at $i$ can be expressed by (4): 


$$
B_{i}(\boldsymbol{y})=\sum_{\substack{j \in J \\ i=k(\boldsymbol{y}, j)}} b_{j} .
$$

If $N(\boldsymbol{y})$ denotes the cardinality of the set $I_{1}(\boldsymbol{y})=$ $\left\{i \in I: y_{i} \geq 1\right\}$, then a mathematical model of the problem with the second objective can be formulated as (5):

$$
\min \left\{\sum_{i \in I_{1}(\boldsymbol{y})} \frac{\left(B_{i}(\boldsymbol{y})\right)^{2}}{y_{i} \cdot N(\boldsymbol{y})}-\frac{\sum_{j \in J} b_{j}}{p \cdot N(\boldsymbol{y})}: \boldsymbol{y} \in Q\right\} .
$$

The common approach to the problems with the two objectives consists in adjusting the first objective to linear form by introducing so called allocation variables and in fixing an upper bound of each $B_{i}(\boldsymbol{y})$ instead of dealing with the nonlinear expression of dispersion, which corresponds to the second objective. Such approach leads to formulation of a capacitated location problem, where capacity limit $a$ on volume of population serviced by one facility is introduced to formulate the service center capacity of the individual service centers. To complete the location model, the allocation variables $z_{i j}$ are introduced, to model the decision on assignment of the demand of users located at $j$ to the possible center location $i$. Variable $z_{i j}$ takes value of the portion of demand at $j$ serviced by facilities located at $i$.

After these preliminaries, a model of the problem can be written as:

Minimize:

$$
\sum_{i \in I} \sum_{j \in J} d_{i j} \cdot b_{j} \cdot z_{i j}
$$

subject to:

$$
\begin{aligned}
& \sum_{i \in I} z_{i j}=1 \text { for } j \in J \\
& z_{i j} \leq y_{i} \text { for } i \in I, j \in J \\
& \sum_{j \in J} b_{j} \cdot z_{i j} \leq a \cdot y_{i} \text { for } i \in I \\
& \sum_{i \in I} y_{i} \leq p ; \\
& y_{i} \in Z_{0}^{+} \text {for } i \in I \\
& z_{i j} \geq 0 \text { for } i \in I, j \in J .
\end{aligned}
$$

The $p$-median objective function (6) is represented by sum of distances from individual users to the service center location assigned to a user by allocation variables $z_{i j}$. The constraints (7) allocate the users sharing location $j$ to possible service center locations, while constraints (8) ensure that a service center location is equipped with a facility whenever a user is assigned to the location. Constraints (9) describe relationship between the number of users assigned to service center location $i$ and the total capacity of the center. Constraint (10) limits the total number of deployed facilities and obligatory constraints (11) and (12) specify domains of the decision variables. The resulting model is similar to the capacitated $p$-median problem, which is known to be Non-deterministic Polynomial-time (NP)-hard. As a consequence, it was found that the problem can be hardly solved to optimality in acceptable time even for moderate sized problems (Janáček et al. 2010; Gabrisova, Janacek 2015).

In addition, we show that the capacity constraints (9) imposed on problem solutions deform the users' clusters and thus the objective function does not correspond to the real objective function, which follows the natural cluster forming. This represents the basic discrepancy between capacitated location model and the studied emergency system. The model inconsistency will be demonstrated by the following counterexample, where we consider the network depicted in Figure.

The network consists of two sets of nodes depicted as squares or circles, where the squares represent possible center locations and the circles represent individual users. Lengths of the edges are attached to the associated links

\begin{tabular}{|c|c|c|c|}
\hline (1) & (2) & (3) & (4) \\
\hline (1) & 1 & 1 & 8 \\
\hline 2 & 6 & 4 & 3 \\
\hline 3 & 9 & 7 & 1 \\
\hline
\end{tabular}
connecting pairs of nodes.

The matrix of distances among possible center locations and users is plotted in Table 1.

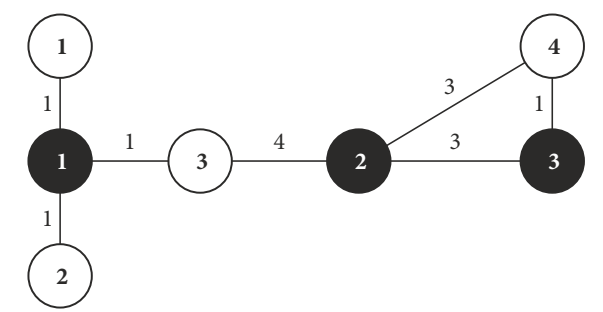

Figure. Network connecting the possible center "@" locations and individual users " $\bigcirc$ "

Table 1. The matrix of distances among possible center " locations and users "O"

Contrary to the natural clusters of the optimal solution of (6)-(12), the solution, which locates the two facilities at possible center location 1 and 3 , has the objective function value of 4 having broken the capacity constraint by the same way as the natural clusters of the optimal solution of (6)-(12). It follows that the model (6)-(12) of capacitated location problem does not describe the original problem with the two objectives. That is why; we suggest several different methods to solve the two - objective problem and explore their characteristics of the methods.

\section{Relaxation and decomposition heuristics for fairer emergency system design}

Within this section, we present and compare four heuristic methods, which can be used to design fairer emergency service system. The first method called "Partial capacity relaxation" is obtained by straightforward standard way, 
where the capacitated problem formulation (6)-(12) is employed and the capacity limit $a$ is relaxed to $a+a_{+}(\mathrm{Ga}-$ brisova, Janacek 2015). This approach belongs to standard ones, but this approach prevents forming natural clusters due to restricted capacity. The next approach called "decomposition method" (Decomp) was introduced first time in research by Gabrisova and Janacek (2015), as the method, which does not deform the natural clusters by the capacity limits, but it allocates resources so that demands of naturally formed clusters were covered as evenly as possible. For sake of clarity, we present a concise description of the method.

The third method uses the transportation problem formulation for step-by-step diminishing the set of supplying centers until cardinality of the set is less than or equal to the number $p$ and, subsequently, it reduces the number of used facilities at the value $p$. The fourth method uses optimal solution of the $p$-median instance to reduce the set of possible center locations and, then it determines the number of necessary facilities for each center so that the volumes of natural clusters are covered. Then, the method reduces the number of determined facilities by a greedy heuristic to the number $p$. These methods are described in details in the remainder of this section.

\subsection{Partial capacity relaxation}

The first approach is based on partial relaxation of the capacity constraints (9). The relaxation consists of increasing the capacity $a$ about some value $a_{+}$. Our preliminary research (Janáček, Gábrišová 2009; Gabrisova, Janacek 2015) showed that the convergence of the solving process of the problem (6)-(12) can be considerable improved, if the original service center capacity is relaxed. The value $a_{+}$is proportional to the value $a$. New form of the constraints (13) follows:

$$
\sum_{j \in J} b_{j} \cdot z_{i j} \leq\left(a+a_{+}\right) y_{i} \text { for } i \in I \text {. }
$$

This relaxation enables to obtain a slightly infeasible solution in acceptable computational time. This approach will be denoted by the title Relax.

\subsection{Decomposition method}

To make the descriptions of algorithms in current Section more readable, we introduce the following denotation. We define function $p M(S, p)$, which computes optimal solution $(y, z)$ of the $p$-median problem (6)-(8), (10)-(12) for the above defined input structures $J, I, b_{j}$ for $j \in J$ and $d_{i j}$ for $i \in I, j \in J$ and $S \subseteq I$, where $S$ is considered to be a reduced set of possible center locations. The output of the function $p M(S, p)$ is formed by pair $\left(B, I_{1}\right)$, where $I_{1}$ corresponds to $I_{1}(\boldsymbol{y})$ and $\boldsymbol{B}$ is a system $\left\{B_{i}: i \in I_{1}\right\}$. In addition to the above mentioned input structures, we consider a next input parameter $a$ as capacity of facility. We assume that $p \cdot a$ is greater than or equal to the sum of all $b_{j}$. The heuristic method denoted as Decomp solves the fol- lowing sub-problems successively in the following four phases according to research by Gabrisova and Janacek (2015).

The first phase - solving of $p$-median problem:

Define $\left(\boldsymbol{B}, I_{1}\right)=p M(I, p)$.

The second phase - allocation of facilities located in the first phase:

Divide the set $I_{1}$ into two sub-sets $I_{1}^{-}=\left\{i \in I_{1}: B_{i} \leq a\right\}$, $I_{1}^{+}=\left\{i \in I_{1}: B_{i}>a\right\}$ and denote $B_{i}^{-}=B_{i}$ for $i \in I_{1}^{-}$and $B_{i}^{+}=B_{i}-a$ for $i \in I_{1}^{+}$.

To model the decisions on reallocating the capacities of the closed centers, we introduce the variable $x_{k j} \in\{0,1\}$ for each pair $k, j$, where $k \in I_{1}^{-}, j \in I_{1}^{+}$. The variable $x_{k j}$ takes the value of 1 , if the facility $k$ is to be closed and its capacity $a$ is to be moved to the center $j$, otherwise the variable takes the value of 0 . An auxiliary nonnegative variable $u_{j}$ is introduced to model volume of the "revenue" at the center $j$.

Solve the following problem:

Maximize:

$\sum_{j \in I_{1}^{+}} u_{j}-\sum_{j \in I_{1}^{+}} \sum_{k \in I_{1}^{-}} B_{k}^{-} \cdot x_{k j}$

subject to:

$$
\begin{aligned}
& \sum_{j \in I_{1}^{+}} x_{k j} \leq 1 \text { for } k \in I_{1}^{-} ; \\
& u_{j} \leq B_{j}^{+} \text {for } j \in I_{1}^{+} ; \\
& u_{j} \leq a \sum_{k \in I_{1}^{-}} x_{k j} \text { for } j \in I_{1}^{+} ; \\
& x_{k j} \in\{0,1\} \text { for } k \in I_{1}^{-}, j \in I_{1}^{+} ; \\
& u_{j} \geq 0 \text { for } j \in I_{1}^{+} .
\end{aligned}
$$

The expression (14) represents the maximized "profitability" subject to the system of constraints, where constraints (15) ensure that unutilized center capacity can be reallocated at most to one overloaded center. The constraints (16) and (17) assure that the "revenue" at overloaded center $j$ cannot exceed the associated surplus and also the total capacity reallocated to the center $j$ cannot be surpassed.

The third phase - determination of the reduced number $p_{1}$ :

$$
\begin{aligned}
& \text { set } p_{1}=p-\sum_{j \in I_{1}^{+}} \sum_{k \in I_{1}^{-}} x_{k j} ; \\
& \text { redefine }\left(B, I_{1}\right)=p M\left(I, p_{1}\right) .
\end{aligned}
$$

The fourth phase - final facility reallocation:

The set $I_{1}$ consists of $p_{1}$ locations and we assume that each center $i \in I_{1}$ is equipped by one facility with capacity $a$. Remaining free $p-p_{1}$ facilities are allocated accordingly to the further procedure. We introduce an integer variable $x_{i}$ for each $i \in I_{1}$, to model the number of additional facilities assigned to the center $i$. 
Additionally, we introduce an auxiliary variable $h$, to express the lower bound of all ratios of allocated capacity to surplus at a given center.

Solve the following problem: maximize:

$h$

subject to:

$$
\begin{aligned}
& x_{i}+1 \geq B_{i} \cdot h \text { for } i \in I_{1} ; \\
& \sum_{i \in I^{*}} x_{i} \leq p-p_{1} ; \\
& x_{i} \in Z_{0}^{+} \text {for } i \in I_{1} .
\end{aligned}
$$

The resulting solution assigns the remaining facilities to the centers, and thus $y_{i}=x_{i}+1$ facilities will be located at center $i \in I_{1}$.

\subsection{Transportation problem}

To make the descriptions of presented algorithm more concise, we define function $T P(S, A)$, which gives an optimal solution $x$ of the non-balanced transportation problem for the following input structures. In the transportation problem, there are used structures $J$, as mentioned above, where $J$ is set of customers, $b_{j}$ corresponds to the demand of customer $j$ and $d_{i j}$ is used here as unit cost for transportation of one unit from location $i$ to location $j$. The set $S \subseteq I$ is considered to be the set of suppliers with list $A$ of supplies, where $A=\left\{a_{i}: i \in S\right\}$. The output of the function $T P(S, A)$, is formed by system $\boldsymbol{B}=\left\{B_{i}: i \in S\right\}$, where $B_{i}$ corresponds to the volume transported from supplier $i$ to all customers.

The objective of the problem is to satisfy all customers' demands from the available supply so that the total cost of transportation proportional to distances is minimal. It is considered that sum of supplies exceeds the sum of demands.

The optimal solution of the above-defined transportation problem is usually described by set of values $x_{i j}$ for $i \in S$ and $j \in J$, where the value $x_{i j}$ denotes the volume of goods or material transported from the supplier $i$ to the customer $j$. The result of the transportation problem is denoted as $\boldsymbol{B}=T P(S, A)$, where value $B_{i}$ is defined by the equation:

$$
B_{i}=\sum_{j \in J} x_{i j} \text { for } i \in S
$$

In addition to the above mentioned input structures, we consider a next input parameter $a$ as capacity of facility in the suggested method and $p$, which limits the number of located facilities.

The suggested method consists of two phases, where the first phase reduces the cardinality of the set $S$ of centers to the number of $p$ and the second phase adjusts the number of located facilities at the number of $p$.

\section{The first phase:}

$$
\begin{aligned}
& \text { 0. Define } Q=\sum_{j \in J} b_{j}, w=\left\lceil\frac{Q}{p}\right\rceil, S=I, a_{i}=Q \text { for all } \\
& i \in S
\end{aligned}
$$

1. Compute $\boldsymbol{B}=T P(S, A)$;

2. If $|S|>p$, then determine $i^{*}=\operatorname{argmin}\left\{B_{i}: i \in S, B_{i}<w\right\}$ and update $S=S-\left\{i^{*}\right\}$ and go to step 1 .

Otherwise, terminate the first phase.

\section{The second phase:}

0. Define $e_{i}=\left\lceil\frac{B_{i}}{w}\right\rceil$ and $\underline{e}_{i}=\left\lfloor\frac{B_{i}}{w}\right\rfloor$ for $i \in S$. Then determine lists $A=\left\{a_{i}: i \in S\right\}$ and $A=\left\{a_{i}: i \in S\right\}$ by $a_{i}=e_{i} \cdot w$ and $\underline{a}_{i}=\underline{e}_{i} \cdot w$ for $i \in S$. Set $q=\sum_{i \in S} e_{i}$;

1. If $q>p$, then determine $i^{*}=\operatorname{argmin}\left\{B_{i}-\underline{a}_{i}: i \in S, B_{i}>\underline{a}_{i}\right\}$ and adjust $a_{i}^{*}=\underline{a}_{i}$ and if $\underline{a}_{i}^{*}=0$, then update $S=S-\left\{i^{*}\right\}$.

Otherwise, terminate the second phase.

2. Compute $\boldsymbol{B}=T P(S, A)$.

Update $e_{i}, \underline{e}_{i}, a_{i}, \underline{a}_{i}$ and $q$ accordingly to the rules from the step 0 and go to step 1 .

After the both phases have been performed, we obtain the set $S$ of service center locations, where each center $i$ should be equipped with $e_{i}$ facilities.

\section{4. $p$-median problem}

Capacitated emergency public service system design employing $p$-median problem formulation uses optimal solution of the $p$-median instance to reduce the set of possible center locations and, then the method locates the remaining non-located facilities by a greedy heuristic. The suggested approach can be formulated as the following algorithm:

0. Initialize $S=I, Q=\sum_{j \in J} b_{j}, w=\left\lfloor\frac{Q}{p}\right\rfloor$;

1. Compute $\left(\boldsymbol{B}, I_{1}\right)=p M(S, p)$;

2. Determine $S=I_{1}, e_{i}=\left\lceil\frac{B_{i}}{w}\right\rceil, \underline{e}_{i}=\left\lfloor\frac{B_{i}}{w}\right\rfloor$ for $i \in S$. Then determine lists $A=\left\{a_{i}: i \in S\right\}$ and $A=\left\{a_{i}: i \in S\right\}$ by $a_{i}=e_{i} \cdot w$ and $\underline{a}_{i}=\underline{e}_{i} \cdot w$ for $i \in S$. Set $\underline{q}=\sum_{i \in S} \underline{e}_{i}$.

Number $e_{i}$ denotes the number of facilities, which are to be located at center location $i$. Difference $p-\underline{q}$ corresponds to the number of non-located facilities.

3. Compute priority coefficients $f_{i}=\frac{B_{i}-a_{i}}{w \cdot e_{i}}$ for $i \in S$ and locate the $p-\underline{q}$ remaining facilities at $p-\underline{q}$ locations with the highest priority coefficients $f_{i}$.

\section{Numerical experiments}

The goal of the numerical experiments is comparison of the suggested methods, especially we want to find a relation between the classical min-sum objective function and some measure of cluster fairness, which is here expressed 
by standard deviation of the demands served by individual facilities.

To compare the approaches mentioned in the Section 3, the following numerical experiments were performed. The used benchmarks were derived from real emergency health care system, which was originally designed for eight self-governing regions of Slovak Republic. The original solutions are referred as Original. The instances are denoted so that they correspond to the administrative organization of Slovakia (Bratislava - BA, Banská Bystrica - BB, Košice - KE, Nitra - NR, Prešov - PO, Trenčín - TN, Trnava - TT and Žilina - ZA). The number of inhabitants at users' location $j$ (i.e. town, village, etc.) is rounded to hundreds and denoted as $b_{j}$. The facility mentioned in the previous Sections corresponds to one ambulance vehicle. The capacity limit of the facility was set at the value of the number of inhabitants, which falls upon one ambulance vehicle, i.e. $a=\sum_{j \in J} \frac{b_{j}}{p}$.

The number $p$ of deployed facilities corresponds to the real number of ambulance vehicles located in the given self-governing region. To be able to evaluate the results of the approaches and the current emergency system, we take into account that the result of each of the approaches can be described by a vector $y$, which consists of integer components $y_{i}$ for $i \in I$. The value of $y_{i}$ gives the number of facilities, which should be located at the location $i$. For each solution $y, I_{1}(y)$ denotes the set of located centers, i.e. $I_{1}(\boldsymbol{y})=\left\{i \in I: y_{i} \geq 1\right\}$. Furthermore, $J_{i}(\boldsymbol{y})$ denotes the set of user locations, which are assigned to the center location $i$. The cluster $J_{i}(\boldsymbol{y})$ of the located center $i \in I_{1}(\boldsymbol{y})$ can be defined by the equality $J_{i}(\boldsymbol{y})=\left\{j \in J: d_{i j}=\min \left\{d_{k j}: k \in I_{1}(\boldsymbol{y})\right\}\right\}$, where $d_{i j}$ is the distance between center $i \in I$ and municipality $j \in J$ for all $i \in I, j \in J$ where $i \neq j$ and $d_{i i}$ is defined as the average distance of inhabitants from center $i$ of the municipality. Then the volume of demands served by center $i$ can be expressed as $B_{i}(\boldsymbol{y})=\sum_{j \in J_{i}(\boldsymbol{y})} b_{j}$ and the part of the volume, which must be served by one facility (ambulance) located at that center, is computed as $\frac{B_{i}(\boldsymbol{y})}{y_{i}}$. The basic characteristics of used benchmarks (Szendreyová 2015) are described in Table 2 . The column denoted as $|I|$ contains numbers of possible service center locations. This number is equal to the number of the user's locations. The column denoted by $p$ contains maximal number of ambulance vehicles, which are to be deployed. The column "Inhabitants" gives number of inhabitants of the self-governing region in hundreds. This number is considered as the total demand of the region. The column " $a$ " contains the portion of demand, which must be served by one ambulance on average.

The original design and the subsequent four designs obtained by the mentioned approaches of the emergency service system design were evaluated and obtained char-
Table 2. The description of used benchmarks (Gabrisova, Janacek 2015)

\begin{tabular}{|c|c|c|c|c|c|c|}
\hline Region & $|I|$ & $|J|$ & $p$ & Inhabitants & $a$ & $a+a_{+}$ \\
\hline BA & 87 & 87 & 25 & 6063 & 243 & 264 \\
\hline TT & 249 & 249 & 22 & 5552 & 253 & 275 \\
\hline TN & 276 & 276 & 26 & 5942 & 229 & 248 \\
\hline NR & 350 & 350 & 36 & 6896 & 192 & 208 \\
\hline ZA & 315 & 315 & 36 & 6896 & 192 & 208 \\
\hline BB & 515 & 515 & 46 & 6601 & 144 & 156 \\
\hline PO & 664 & 664 & 44 & 8158 & 186 & 202 \\
\hline KE & 460 & 460 & 38 & 7930 & 209 & 227 \\
\hline
\end{tabular}

acteristics of the designs are plotted in the following series of seven tables. The tables correspond with the individual self-governing regions accordingly to the list presented in Tables 3-10.

Each column with exception of " $p$-median" corresponds to one solution of the service system design problem, which was either obtained by some of the suggested methods or represents the current service center deployment.

The following column denotations are used:

- "Original" denotes the current solution of the service system design problem, which can be obtained from (Szendreyová 2015) including the current solution;

- "p-median" corresponds to the reference solution obtained by solving the simple weighted $p$-median problem, which minimizes sum of distances from users' locations to the nearest service center location. The sizes of problem instances follow: the number of non-integer variables is $|I| \times|J|$, the number of integer variables is $|I|$ and the number of structural constraints is $|J|+|I| \times|J|+1$;

- "Relax" denotes the solution, which was obtained by solving the partial relaxation of the capacitated location problem (6)-(12). The sizes of problem instances follow: the number of non-integer variables is $|I| \times|J|$, the number of integer variables is $|I|$ and the number of structural constraints is $|J|+|I| \times|J|+|I|+1$;

- "Decomp" corresponds with the solution obtained by the decomposition method described in Subsection 3.2;

- " $T P$ ” denotes the solution obtained by newly suggested method based on iterative process, which solves a transportation problem at each iteration of the process. The maximal sizes of problem instances follow: the number of non-integer variables is $|I| \times|J|$ and the number of structural constraints is $|J|+|I|-1$;

- " $p M$ " denotes solution obtained by the two-phase method, which solves the $p$-median problem first, to obtain a reduced set of potential service centers and then it performs further reduction of this set to balance demand volume of the individual facilities. 
The maximal sizes of problem instances follow: the number of non-integer variables is $|I| \times|J|$, the number of integer variables is $|I|$ and the number of structural constraints is $|J|+|I| \times|J|+1$.

The suggested approaches were implemented in the visual development environment Xpress-IVE (https://www. fico.com/en/products/fico-xpress-optimization) using solver Xpress-Optimizer v7.3. The experiments were performed on a personal computer equipped with the Intel Core i7 processor with $1.60 \mathrm{GHz}$ and $8 \mathrm{~GB}$ of RAM.

The particular rows of the tables correspond with individual characteristics of the solutions obtained by the tested methods. The following denotations of the observed characteristics are used:

- "OF" denotes objective function value of the solution computed in accordance with (25):

$\sum_{i \in I_{1}(\boldsymbol{y})} \sum_{j \in J_{i}(\boldsymbol{y})} d_{i j} \cdot b_{j} \quad[100$ person $\times \mathrm{km}] ;$

- "noC" denotes the number of deployed service centers; it must be noted that more than one facility can be located at one service center location;

- "StD" denotes standard deviation of demand volumes served by particular facilities from the average demand, which comes upon one facility; the standard deviation was computed in accordance with (26):

$$
S t D=\sqrt{\frac{\sum_{i \in I_{1}(\mathbf{y})}\left(\frac{B_{i}(\boldsymbol{y})}{y_{i}}-a\right)^{2}}{n o C-1}} ;
$$

- "relR" denotes relative range of values $\frac{B_{i}(\boldsymbol{y})}{y_{i}}$ com-
puted in accordance with (27):

$$
\frac{\max \left\{\frac{B_{i}(\boldsymbol{y})}{y_{i}}: i \in I_{1}(\boldsymbol{y})\right\}-\min \left\{\frac{B_{i}(\boldsymbol{y})}{y_{i}}: i \in I_{1}(\boldsymbol{y})\right\}}{a}
$$

- " $c T$ " denotes computational time in seconds consumed by the particular method for obtaining the resulting solution;

- These experiments were performed to find the most suitable method of emergency system design, which provides fair clusters of users, where the demand is equally distributed among located facilities.

Table 3. Comparison of the results for the region BA

\begin{tabular}{|l|c|c|c|c|c|c|}
\hline Characteristic & Original & $p$-median & Relax & Decomp & TP & $\mathrm{pM}$ \\
\hline OF & 21842 & 12834 & 16176 & 16611 & 17405 & 14428 \\
\hline noC & 25 & 25 & 19 & 17 & 17 & 21 \\
\hline$S t D$ & 71 & 222 & 86 & 76 & 64 & 100 \\
\hline$r e l R$ & 1.31 & 4.13 & 1.23 & 1.13 & 1.00 & 1.27 \\
\hline$c T$ & 0 & 0 & 2 & 9 & 5 & 0 \\
\hline
\end{tabular}

Table 4. Comparison of the results for the region TT

\begin{tabular}{|l|c|c|c|c|c|c|}
\hline Characteristic & Original & $p$-median & Relax & Decomp & TP & $\mathrm{pM}$ \\
\hline OF & 31582 & 24935 & 27414 & 29232 & 32434 & 26834 \\
\hline noC & 22 & 22 & 20 & 17 & 16 & 20 \\
\hline StD & 116 & 174 & 87 & 88 & 68 & 116 \\
\hline relR & 1.92 & 3.04 & 1.14 & 1.04 & 0.79 & 1.43 \\
\hline$c T$ & 0 & 0 & 2133 & 142 & 31 & 0 \\
\hline
\end{tabular}

Table 5. Comparison of the results for the region TN

\begin{tabular}{|l|c|c|c|c|c|c|}
\hline Characteristic & Original & $p$-median & Relax & Decomp & TP & $\mathrm{pM}$ \\
\hline OF & 26683 & 22204 & 24518 & 26479 & 29256 & 24665 \\
\hline noC & 26 & 26 & 23 & 19 & 17 & 22 \\
\hline StD & 134 & 166 & 100 & 71 & 56 & 93 \\
\hline relR & 2.12 & 2.68 & 1.52 & 1.04 & 0.92 & 1.46 \\
\hline$c T$ & 0 & 0 & 4010 & 213 & 34 & 0 \\
\hline
\end{tabular}

Table 6. Comparison of the results for the region NR

\begin{tabular}{|l|c|c|c|c|c|c|}
\hline Characteristic & Original & $p$-median & Relax & Decomp & TP & $\mathrm{pM}$ \\
\hline OF & 38831 & 28866 & 33930 & 34758 & 36223 & 34111 \\
\hline noC & 36 & 36 & 29 & 26 & 26 & 28 \\
\hline StD & 77 & 171 & 65 & 51 & 47 & 78 \\
\hline$r e l R$ & 1.34 & 4.17 & 1.44 & 0.87 & 0.79 & 1.95 \\
\hline$c T$ & 0 & 0 & 122 & 227 & 71 & 0 \\
\hline
\end{tabular}

Table 7. Comparison of the results for the region $\mathrm{ZA}$

\begin{tabular}{|l|c|c|c|c|c|c|}
\hline Characteristic & Original & $p$-median & Relax & Decomp & TP & $\mathrm{pM}$ \\
\hline OF & 31955 & 25433 & 30410 & 32411 & 36013 & 32149 \\
\hline noC & 29 & 36 & 29 & 24 & 22 & 26 \\
\hline StD & 105 & 188 & 66 & 63 & 41 & 70 \\
\hline relR & 1.69 & 4.84 & 1.17 & 1.08 & 0.88 & 1.49 \\
\hline$c T$ & & & 5888 & 266 & 62 & \\
\hline
\end{tabular}

Table 8. Comparison of the results for the region $\mathrm{BB}$

\begin{tabular}{|l|c|c|c|c|c|c|}
\hline Characteristic & Original & $p$-median & Relax & Decomp & TP & $\mathrm{pM}$ \\
\hline OF & 32476 & 25748 & 31127 & 31548 & 34906 & 31461 \\
\hline noC & 46 & 46 & 35 & 33 & 31 & 35 \\
\hline StD & 76 & 147 & 46 & 42 & 33 & 58 \\
\hline relR & 2.53 & 6.00 & 1.16 & 1.06 & 0.95 & 1.60 \\
\hline$c T$ & 0 & 0 & 2228 & 963 & 204 & 0 \\
\hline
\end{tabular}


Table 9. Comparison of the results for the region PO

\begin{tabular}{|l|c|c|c|c|c|c|}
\hline Characteristic & Original & $p$-median & Relax & Decomp & TP & pM \\
\hline OF & 42740 & 32679 & 38621 & 41126 & 45277 & 38980 \\
\hline$n o C$ & 44 & 44 & 36 & 29 & 28 & 33 \\
\hline$S t D$ & 85 & 188 & 74 & 51 & 50 & 65 \\
\hline$r e l R$ & 1.99 & 5.39 & 1.80 & 0.92 & 0.98 & 1.32 \\
\hline$c T$ & 0 & 0 & 3621 & 2268 & 353 & 0 \\
\hline
\end{tabular}

Table 10. Comparison of the results for the region $\mathrm{KE}$

\begin{tabular}{|l|c|c|c|c|c|c|}
\hline Characteristic & Original & P-median & Relax & Decomp & TP & pM \\
\hline OF & 36363 & 27853 & 34232 & 37150 & 39713 & 34685 \\
\hline noC & 38 & 38 & 35 & 25 & 27 & 29 \\
\hline StD & 127 & 206 & 108 & 69 & 53 & 86 \\
\hline$r e l R$ & 3.38 & 4.72 & 2.56 & 1.04 & 0.83 & 1.64 \\
\hline$c T$ & 0 & 0 & 3613 & 984 & 132 & 0 \\
\hline
\end{tabular}

The method "TP" proved to be the winner of the comparison from the point of both standard deviation and relative range, which were the main criteria. The method " $T P$ " is followed by the method "Decomp". Nevertheless, the method " $T P$ " loses as concerns the objective function value, where the objective function value of each "TP" solution is considerably higher than the value of solution obtained by method "Decomp" In addition, the objective values of " $T P$ " solutions are bigger than the values of original solutions in six from eight cases, whereas the "Decomp" method obtained only two from eight solutions with worse objective function value. As concerns the computational time, the both discussed winners yielded the solution in acceptable time. Even if the " $T P$ " method proved to be in order faster, it must be noted that the speed of computational process convergence is influenced by proper setting of parameters. The computational time reported in the tables corresponds to the best experimented setting. That is why, the two methods "Decomp" and "TP" should be taken into account for the next research. As concerns the remaining methods, it can be noted that the "Relax" failed due to very slow convergence to the resulting solution and the " $T P$ " method failed in the competition, when standard deviation is considered.

\section{Conclusions}

We have developed and compared four approaches to fair emergency service system design to mitigate frequency of the events, where a user demand must be served from a service center, which is more distant than the nearest one.

The principle of the mitigation is based on producing such service center deployment that the resulting natural clusters of users have equal volumes of demand per one facility, what can be denoted as a kind of fairness.
The approaches have been suggested so that they are implementable on a commercial IP-solver, what enables compressing the long terms of a software development, when an emergency public service system is designed.

The performed comparison can be concluded by the choice of two approaches denoted as "Decomp" and "TP" for the next usage and development.

The further research in this field of fair emergency system design will be aimed at possible merging of the two chosen approaches. In addition, some other kinds of the cluster fairness can be included in the future research.

The workload of a cluster defined as a sum of users' distances to the nearest center multiplied by users' demand volumes can be used instead of simple sum of the demand volumes.

In addition, combined criteria imposing the maximal distance limit on distance between user and the nearest located center deserve attention of researchers.

\section{Acknowledgements}

This work was supported by the research grants VEGA 1/0518/15 "Resilient rescue systems with uncertain accessibility of service" and APVV-15-0179 "Reliability of emergency systems on infrastructure with uncertain functionality of critical elements".

We would also like to thank to "Centre of excellence for systems and services of intelligent transport" (ITMS 26220120050) for built up the infrastructure, which was used.

\section{References}

Avella, P.; Sassano, A.; Vassil'ev, I. 2007. Computational study of large-scale $p$-median problems, Mathematical Programming 109(1): 89-114. https://doi.org/10.1007/s10107-005-0700-6

Bertsimas, D.; Farias, V. F.; Trichakis, N. 2011. The price of fairness, Operations Research 59(1): 17-31.

https://doi.org/10.1287/opre.1100.0865

Brotcorne, L.; Laporte, G.; Semet, F. 2003. Ambulance location and relocation models, European Journal of Operational Research 147(3): 451-463.

https://doi.org/10.1016/S0377-2217(02)00364-8

Buzna, L.; Koháni, M.; Janáček, J. 2014. An approximation algorithm for the facility location problem with lexicographic minimax objective, Journal of Applied Mathematics 2014: 562373. https://doi.org/10.1155/2014/562373

Chanta, S.; Mayorga, M. E.; McLay, L. A. 2014. Improving emergency service in rural areas: a bi-objective covering location model for EMS systems, Annals of Operations Research 221(1): 133-159. https://doi.org/10.1007/s10479-011-0972-6

Doerner, K. F.; Gutjahr, W. J.; Hartl, R. F.; Karall, M.; Reimann, M. 2005. Heuristic solution of an extended double-coverage ambulance location problem for Austria, Central European Journal of Operations Research 13(4): 325-340.

Erlenkotter, D. 1978. A Dual-based procedure for uncapacitated facility location, Operations Research 26(6): 992-1009. https://doi.org/10.1287/opre.26.6.992

Gabrisova, L.; Janacek, J. 2015. Design of capacitated emergency service system, Communications: Scientific Letters of the University of Žilina 17(2): 42-48. 
García, S.; Labbé, M.; Marín, A. 2011. Solving large p-median problems with a radius formulation, INFORMS Journal on Computing 23(4): 546-556. https://doi.org/10.1287/ijoc.1100.0418

Holmberg, K.; Rönnqvist, M.; Yuan, D. 1999. An exact algorithm for the capacitated facility location problems with single sourcing, European Journal of Operational Research 113(3): 544-559. https://doi.org/10.1016/S0377-2217(98)00008-3

Ingolfsson, A.; Budge, S.; Erkut, E. 2008. Optimal ambulance location with random delays and travel times, Health Care Management Science 11(3): 262-274.

https://doi.org/10.1007/s10729-007-9048-1

Janáček, J. 2008. Použití komerčního IP-solveru pro řešení umistovacích úloh, Perner's Contacts 3(5): 119-124 (in Czech).

Janáček, J.; Buzna, L. 2008. An acceleration of Erlenkotter-Körkel's algorithms for the uncapacitated facility location problem, Annals of Operations Research 164(1): 97-109. https://doi.org/10.1007/s10479-008-0343-0

Janáček, J.; Gábrišová, L. 2009. A two-phase method for the capacitated facility problem of compact customer sub-sets, Transport 24(4): 274-282.

https://doi.org/10.3846/1648-4142.2009.24.274-282

Janáček, J.; Janáčková, M.; Szendreyová, A.; Gábrišová, L.; Koháni, M.; Jánošíková, L. 2010. Navrhovanie územne rozlahlých obslužných systémov. Žilinská univerzita. 404 s. (in Slovak).

Janacek, J.; Kvet, M. 2014. Relevant network distances for approximate approach to large $p$-median problems, in Operations Research Proceedings 2012: Selected Papers of the International Annual Conference of the German Operations Research Society (GOR), 5-7 September 2012, Hannover, Germany, 123-128. https://doi.org/10.1007/978-3-319-00795-3_18

Janacek, J.; Kvet, M. 2012. Sequential zone adjustment for approximate solving of large $p$-median problems, in Operations Research Proceedings 2011: Selected Papers of the International Conference on Operations Research (OR 2011), 30 August-2 September 2011, Zurich, Switzerland, 269-274. https://doi.org/10.1007/978-3-642-29210-1_43

Jánošíková, L. 2007. Emergency medical service planning, Communications: Scientific Letters of the University of Žilina 9(2): 64-68.

Jánošíková, L.; Žarnay, M. 2014. Location of emergency stations as the capacitated $p$-median problem, in Proceedings of the International Scientific Conference "Quantitative Methods in Economics: Multiple Criteria Decision Making XVII", 28-30 May 2014, Virt, Slovakia, 116-122.

Körkel, M. 1989. On the exact solution of large-scale simple plant location problems, European Journal of Operational Research 39(2): 157-173.

https://doi.org/10.1016/0377-2217(89)90189-6

Kvet, M.; Janáček, J. 2015. Semi-fair deployment of the service centers in a transportation network, in SOR'15: Proceedings of the 13th International Symposium on Operations Research, 23-25 September 2015, Bled, Slovenia, 458-463.

Marianov, V.; Serra, D. 2002. Location problems in the public sector, in Z. Drezner, H. W. Hamacher (Eds.). Facility Location: Applications and Theory, 119-150.

Marsh, M. T.; Schilling, D. A. 1994. Equity measurement in facility location analysis: a review and framework, European Journal of Operational Research 74(1): 1-17. https://doi.org/10.1016/0377-2217(94)90200-3

Ogryczak, W.; Śliwiński, T. 2006. On direct methods for lexicographic min-max optimization, Lecture Notes in Computer Science 3982: 802-811. https://doi.org/10.1007/11751595_85
Pirkul, H.; Schilling, D. 1989. The capacitated maximal covering location problem with backup service, Annals of Operations Research 18(1): 141-154. https://doi.org/10.1007/BF02097800

Szendreyová, B. 2015. Benchmarks from Slovak Road Network. Available from Internet: http://frdsa.fri.uniza.sk/ betka/BenchmarksSR.html 